later, as is shown in $B . \quad C$ is a reproduction of a photograph of another tube through which current has passed for five minutes. The region of high colloid concentration can now be seen extending from the anode to the deposited carbon. Soon after this phase, the region of high colloid concentration assumed an almost rope-like appearance, connecting anode and cathode. Dendrite growth then became extremely rapid and in a few seconds the colloidal mass was reduced to carbon. $D$ shows the final phase; some of the deposited carbon has floated to the top of the tube. During the whole process the potential across the tube did not vary by more than 20 volts, nor did the current exceed more than a few milliamperes. A few small bubbles of gas were liberated at both electrodes during the experiment. $E$ shows carbon dendrites which have been formed in a vertical tube.

A
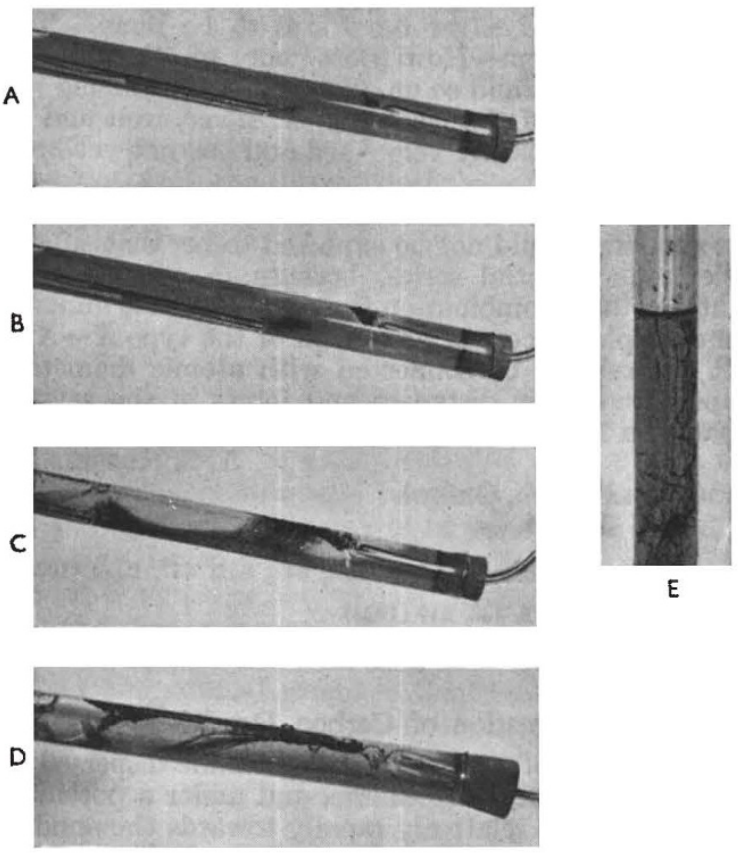

FIG. 1.

It is remarkable that in spite of the high repulsive force which the cathode must exert on the graphitic oxide particles, the latter can remain for a sufficiently long period in contact with the electrode for reduction to occur and for the resulting carbon to attach itself to the electrode. A more detailed examination of the phenomenon is being made.

Armstrong College, Newcastle-upon-Tyne.

1 H. Thiele, Z. anorg. Chem., 190, 145 (1930); Koll. Z., 56, 129 (1931).

\section{A New Process of Negative Ion Formation}

WHEN a stream of electrons is passed through a monatomic gas, negative ions are supposed to be formed by direct electron attachment to atoms of the gas with radiation of the excess energy. When the gas is molecular, the process generally presumed to occur is simultaneous dissociation of the molecule and attachment of the electron to one of the products of dissociation, the energy of the electron in excess of that necessary for dissociation together with the

\begin{tabular}{|l|l||r|c|}
\hline \multicolumn{1}{|c||}{ Process } & $\begin{array}{c}\text { Probability } \\
\times 10^{4}\end{array}$ & Process & $\begin{array}{c}\text { Probability } \\
\times 10^{4}\end{array}$ \\
\hline $\mathrm{Hg}^{+} \rightarrow \mathrm{Hg}^{-}$ & 6.4 & $\mathrm{CO}_{2}^{+} \rightarrow \mathrm{CO}^{-}$ & 2.31 \\
$\mathrm{H}^{2} \rightarrow \mathrm{H}^{-}$ & 0.104 & $\mathrm{CO}^{+}+\mathrm{CO}^{+} \rightarrow \mathrm{CO}^{-}$ & 10.8 \\
$\mathrm{~N}^{+} \rightarrow \mathrm{N}^{-}$ & 1.07 & $\mathrm{CO}^{+} \rightarrow \mathrm{O}_{2}^{-}$ & 2.51 \\
$\mathrm{O}^{+} \rightarrow \mathrm{O}^{-}$ & 0.42 & $\mathrm{CO}_{2}^{+}+\mathrm{CO}^{+} \rightarrow \mathrm{O}^{-}$ & 3.37 \\
$\mathrm{O}^{+} \rightarrow \mathrm{O}^{-}$ & 1.10 & $\mathrm{CO}^{+}+\mathrm{CO}^{+} \rightarrow \mathrm{C}^{-}$ & 0.1 \\
\hline
\end{tabular}

electron affinity of the atom being carried away in kinetic form by the products of dissociation.

I have made an investigation of negative ion formation in mercury vapour, hydrogen, nitrogen, oxygen and carbon dioxide, and I find that the large majority, if not all, the negative ions detected are formed by a new process, and not by either of the processes mentioned above. The negative ions are formed from positive ions which extract two electrons from any negatively charged electrode, including the filament, to which they are driven. This process is energetically possible provided the sum of the ionization potential of the positive ion and the electron affinity of the negative ion that is formed is greater than twice the work function of the surface upon which the conversion takes place. The excess energy is probably dissipated by a collision of the second kind with an electron or atom of the surface.

Sometimes the positive ion is dissociated by its collision with the surface. The negative ion then formed is one of the products of dissociation. For example, the negative ions detected in carbon dioxide are $\mathrm{CO}_{2}^{-}, \mathrm{CO}^{-}, \mathrm{O}_{2}^{-}, \mathrm{O}^{-}$and $\mathrm{C}^{-}$. In oxygen, both $\mathrm{O}_{2}^{-}$and $\mathrm{O}^{-}$are found. Mercury vapour, hydrogen and nitrogen gave only the atomic negative ion.

The probability of conversion of positive ions into negative ions on a nickel surface has been measured as a function of the kinetic energy of the positive ions. In each gas the probability increases as the energy of the positive ion is increased. The probability of conversion of a 180 -volt positive ion into a negative ion is given in the accompanying table in units of $10^{-4}$.

The negative ions come off the surface on which they are formed with a wide range of energy. From the energy distribution curves, values of the mean and the minimum accommodation coefficients for positive ions have been obtained. The energy distribution curve itself represents the probability distribution of the accommodation coefficient.

The fact that positive ions are converted into negative ions on negatively charged electrodes with the comparatively high probability shown in the above table introduces a serious, and hitherto unrecognized, source of error into many experiments, such as the recent work of Davies ${ }^{1}$ on ionization produced by positive ions.

A complete account of this work will be published in the Proceedings of the Royal Society. The work on mercury vapour is already in the press, and should appear shortly.

\section{F. L. Arnot.}

University,

St. Andrews.

'A. C. Davies, Proc. Roy. Soc., A, 155, 123 (1936). 